$\mathbf{m} / \mathbf{S}$

médecine/sciences $1992 ; 8: 420-2$

\title{
LES ARMES PEPTIDIQUES DE LA DÉFENSE ANTIMICROBIENNE : UN CHAMP D'INVESTIGATION EN PLEINE EXPANSION
}

\section{Franc Pattus}

\begin{abstract}
ADRESSE
F. Pattus : directeur de recherche au Cnrs, chef de groupe du département structures biologiques. Laboratoire européen de biologie moléculaire, Meyerhof strasse 1, D6900 Heidelberg, Allemagne.
\end{abstract}

\section{TIRÉS A PART}

F. Pattus.

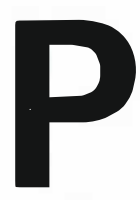

our répondre rapidement et de façon efficace à une invasion microbienne locale lors d'une lésion tissulaire, la réponse immunitaire impliquant les lymphocytes $\mathrm{B}$ et $\mathrm{T}$, et la production d'anticorps assurant la protection à long terme contre les organismes pathogènes, n'est pas suffisamment rapide pour être efficace. Un réseau complexe de systèmes inductibles et non inductibles de défense de l'hôte lui permet de survivre dans un monde truffé de micro-organismes. Chez les mammifères, les cellules phagocytaires les plus abondantes circulant dans le sang, les polynucléaires neutrophiles, possèdent deux mécanismes principaux de défense [1]. La voie dépendante de l'oxygène produit des superoxydes et des agents d'oxydation et de chloration très puissants, tels que l'eau de javel $(\mathrm{HOCl})$ et des chloramines. La voie indépendante de l'oxygène produit des protéines et des peptides antimicrobiens cytotoxiques dans des granules cytoplasmiques qui viennent se déverser dans la vacuole de phagocytose. Chez les insectes, la réponse antibactérienne est un phénomène de réponse humorale inductible qui se caractérise par l'apparition, dans le sang, de peptides à pouvoir antibiotique très efficace ([2] et article de J. Hoffman, p. 432 de ce numéro).

En fait, la production de peptides à pouvoir antibiotique en réponse à l'invasion microbienne ou pour la maintenance d'une niche écologique, se trouve dans l'ensemble du monde vivant. Les bactéries à gram négatif produisent des microcines (de faible poids moléculaire) et des colicines ou bactériocines (de 40000 à 60000 de poids moléculaire) [3]. Les bactéries à gram positif produisent des lantibiotiques et des bactériocines [4]. On retrouve aussi cette classe de peptides dans certaines graines de végétaux et dans la peau des amphibiens (voir l'article de P. Nicolas et al., p. 423 de ce numéro). Chez les mammiferes, outre les neutrophiles, ces peptides sont présents dans des cellules cryptiques de l'intestin et de l'estomac, dans des macrophages pulmo- 
naires et des voies aćriennes. Au cours de ces dernières années, grâce en particulier à l'avènement de la biologie moléculaire, des centaines de séquences peptidiques ont été déterminées, soit par séquençage direct, soit par séquençage des gènes de structure. Il ne se passe pas de semaine sans qu'un nouveau peptide ne soit décrit dans la littérature.

En dépit de ce foisonnement d'information, nos connaissances sur le mode d'action de ces peptides et les mécanismes d'induction de la réponse antimicrobienne sont encore très fragmentaires. On peut toutefois classer les peptides dans différentes superfamilles d'après leur séquence : les motifs peptidiques à trois ponts disulfures; les hélices amphipatiques ; les séquences riches en proline; les séquences riches en glycine.

La famille des défensines, la plus connue, nous fournit un bon exemple de notre ignorance sur leur mode d'action. Les défensines des neutrophiles et les défensines d'insectes sont des peptides de 35 à 50 résidus contenant six cystéines toutes engagées dans des ponts disulfures [2, 5]. Les structures de deux défensives de neutrophile, NP5 et HNP-3 et de deux défensines d'insectes, de phormia et de sarcophaga ont été résolues récemment par RMN et cristallographie aux rayons X ([6-8] et article de J. Hoffmann). Elles ont révélé des différences importantes de structure entre défensines de mammifères et défensines d'insectes. La connexion des ponts disulfures n'est pas la même. Les défensines de mammifères sont riches en feuillets $\beta$ et celles d'insectes contiennent à la fois une hélice $\alpha$ et des brins $\beta$. L'hypothèse selon laquelle les gènes codant pour ces défensines dériveraient d'un ancêtre commun doit donc être exclue.

Curieusement, on retrouve des peptides de même taille à trois ponts disulfure dans les venins de serpent et de scorpion, des neurotoxines et des cardiotoxines [9] et chez l'amibe pathogène Entomoeba histolytica (M. Leippe, communication personnelle). $\mathrm{La}$ structure des défensines de mammifères est voisine, sans être identique, de celles des toxines de venin en feuillet $\beta$. Cette parenté nous est tou$m / s n^{\circ} 5$, vol. 8, mai 92 tefois de peu d'utilité pour émettre une hypothèse sur le monde d'action des défensines. En effet, les toxines $\alpha$ et $\gamma$ de Naja nigricollis présentent une homologie stricte de $42 \%$ et présentent un repliement identique. Cependant leur mode d'action est totalement différent et leur site fonctionnel n'est pas localisé sur la même portion de la molécule [9]. La toxine $\alpha$ se fixe au récepteur de l'acétylcholine, alors que la toxine $\gamma$ agirait au niveau des lipides de la membrane. De même, les défensines d'insectes montrent une connexion des ponts disulfures et une structure semblable à celles des toxines courtes de venin de scorpion auxquelles sont associées plus de sept activités biologiques différentes [9]. Il est donc très probable que, comme pour les toxines de venin, les défensines adoptent des motifs structuraux identiques mais ayant des activités diverses. Il a d'ailleurs été démontré que, outre leur activité de défense contre les microorganismes, plusieurs défensines peuvent être cytotoxiques selon l'activité métabolique de la cellule cible, ou peuvent avoir une activité de type corticostatine (elles interfèrent avec l'effet de l'ACTH sur les cellules corticales), ou sont fortement chimiotactiques pour les monocytes [5].

Comment ces peptides exercent-ils leur activité bactéricide?

Une grande majorité d'entre eux, indépendamment de leur classification selon leur séquence, perméabilise les membranes : externe et interne de $E$. coli et des bactéries à gram positif. In vitro, ils perméabilisent des vésicules lipidiques, et certaines forment des canaux ioniques dépendants du voltage. Dans le cas bien étudié des colicines bactériennes, il a été montré que la perméabilisation de la membrane interne de $E$. coli par un canal ionique était suffisante pour expliquer la grande variété d'effets de cette molécule sur l'état métabolique de la cellule, tels que l'inhibition des transports actifs, l'hydrolyse de l'ATP et l'arrêt de la biosynthèse protéique [3]. Ce type d'activité sur les lipides membranaires expliquerait le spectre assez large d'action de ces peptides dont certains sont ainsi actifs à la fois sur les bactéries à gram positif et négatif, sur certains champignons et certains virus à enveloppe. La structure du site membranolytique, commune à ces peptides, aux cardiotoxines de serpent et aux hémolysines bactériennes, pourrait être une région hydrophobe externe bordée d'une région cationique contenant des lysines et des arginines [10].

Des modes d'actions plus spécifiques doivent expliquer la sélectivité de certains peptides de défense antimicrobienne. Par exemple, il a été montré récemment que l'attacine de Hyalophora cecropia, un peptide de 20000 de poids moléculaire, affecte la perméabilité de la membrane externe d'E.coli en inhibant la synthèse des protéines ompC, ompF, ompA et LamB. Son action se situerait au niveau de la transcription. On ne sait cependant pas si l'attacine est internalisée et peut agir directement sur le promoteur de ces gènes ou bien si son activation est indirecte [11].

Un aspect important $\mathrm{du}$ mode d'action de ces peptides vient du fait qu'ils sont synthétisés avec d'autres peptides et des enzymes telles que le lysozyme. In vivo, ils agissent en synergie. En particulier, les peptides qui perméabilisent la membrane externe de $E$. coli, doivent faciliter l'accès au lysozyme de son substrat, le peptidoglycan. Cette action concertée peut multiplier par un facteur de plusieurs ordres de grandeur l'efficacité des molécules prises isolément comme c'est le cas de la mellitine et de la phospholipase A2 de venin d'abeille.

\section{Comment l'expression génétique des peptides antimicrobiens est-elle contrôlée?}

Les mécanismes de régulation de la biosynthèse de peptides antimicrobiens et de la reconnaissance de l'agression microbienne sont loin d'être élucidés et représentent un défi pour le futur de la recherche dans ce domaine. Chez l'homme, les défensines sont exprimées de façon constitutive par les neutrophiles immatures de la moelle osseuse. Chez les insectes, seules les cellules d'organismes ayant subi une agression transcrivent ces gènes. La nature du signal 
d'agression semble indépendante de la présence de bactéric ou de déterminant bactérien, puisqu'une simple lésion stérile est capable d'induire la transcription coordonnée des gènes immuns [2]. Les possibilités qu'apporte la transgénèse chez la drosophile devraient permettre des avancées importantes dans ce domaine.

\section{Applications potentielles}

La nature se montre d'une efficacité redoutable dans la lutte contre l'invasion microbienne. La panoplie variée d'armes peptidiques à notre disposition doit nous permettre de produire de nouvelles molécules à des fins thérapeutiques et industrielles.

La famille des lantibiotiques produits par les bactéries à gram positif est déjà largement utilisée par l'industrie. Citons pour mémoire la nisine produite par Lactococcus lactis, utilisée depuis des décennies comme conservateur alimentaire, et l'épidermine, utilisée dans le traitement de l'acné [4]. Les défensines de mammiferes sont des sources potenticlles, non seulement de nouveaux antibiotiques contre un large spectre bactérien, mais aussi d'antifongiques. Elles ont aussi une activité antivirale contre les virus à enveloppe.

\section{Conclusion}

Alors que la phagocytose et l'immunité chez les insectes ont été découvertes à la fin du siècle dernier et au début de ce siècle, ce n'est qu'à partir des années 1980 que, grâce aux outils de la biologie moléculaire, nous assistons à une explosion des connaissances sur les mécanismes de défense utilisant des peptides à pouvoir antibiotique. La famille des peptides antimicrobiens est extrêmement diverse du point de vue structural et probablement fonctionnel, mais aussi du point de vue de la biosynthèse et du contrôle de l'expression. La grande variété de séquences peptidiques à la disposition du chercheur va permettre de concevoir de nouvelles générations d'antibiotiques, d'antifongiques et d'antiviraux. Il est toutefois encore nécessaire de faire progresser nos connaissances encore très fragmentaires sur le mode d'action de ces
RÉFÉRENCES

1. Thomas EL, Lehrer Ri, Rest RF. Human neutrophil antimicrobial activity. Rev Infect Dis 1988 ; 10 : S450-6.

2. Dimarcq JL, Zachary D, Hoffmann JA, Hoffmann D, Reichhart JM. Insect immunity : expression of the two major inducible antibacterial peptides in Phormia terranovae. EMBO J 1990 ; 9 : 2507-15.

3. Pattus F, Mascotte D, Wilmsen HU, et al. Colicins : prokaryotic killer pores. Experientia $1990 ; 46: 180-92$.

4. Jung G. Lantibiotics: a survey. In : Jung G, Sahl HG, eds. Nisin and Novel Lantibiotics. Leiden : Escom Sci Publ 1991; $1-34$.

5. Lehrer RI, Ganz T, Selsted ME. Defensins : endogenous antibiotic peptides of animal cells. Cell 1991; 64 : 229-30.

6. Hill CP, Yee J, Selsted ME, Eisenberg D. Crystal structure of defensin HNP-3, an amphiphilic dimer: mechanism of membrane permeabilization. Science 1991 : 251 : 1481-5.

7. Pardi A, Hare DR, Selsted ME, Morisson RD, Bassolino DA, Bach AC II. Solution structures of the rabbit neutrophil defensin NP-5. J Mol Biol 1988; 201: 625-36.

8. Hanzawa H, Shimada I, Kuzuhara T, et al. $1 \mathrm{H}$ nuclear magnetic resonance of the solution conformation of an antibacterial protein, sapecin. FEBS Lett 1990; 269 : 413-20.

9. Bontens F, Roumestand C, Gilquin B, Menez A, Toma F. The refined structure of charybdotoxin reveals the presence of a common structural motif in scorpion toxins and insect defensins. Science 1991; 254: 1521-3.

10. Kini RM, Evans HJ. A common cytolytic region in myotoxins, hemolysins, cardiotoxins and antibacterial peptides. Int $J$ Protein Res $1989 ; 34: 277-86$

11. Carlsson A, Engstrom P, Palva E, Bennich $\mathrm{H}$. Attacin, an antibacterial protein from Hyalophora cecropia, inhibits synthesis of outer membrane proteins in Escherichia coli by interfering with omp gene transcription. Infect Immun 1991; $59: 3040-45$. 